\title{
A new automatic ice-fabric analyzer which uses image-analysis techniques
}

\author{
Wang Yun, Nobuhiko Azuma \\ Nagaoka University of Technology, Kamitomioka-machi 1603-1, Nagaoka, Niigata 940-2188, Japan
}

\begin{abstract}
An automatic ice-fabric analyzer has been developed, which can determine individual $c$-axis orientations by image-analysis techniques. The analyzer consists of four major components: a sample stage, a pair of crossed polaroids, a charge coupled device $(\mathrm{CCD})$ camera and a light source. Both the sample stage and the crossed polaroids can be rotated independently of each other by the stepping motors controlled by a personal computer (PG). Measurements are conducted as follows. An ice thin section is set on the sample stage and then the crossed polaroids are rotated. Thin-section images are recorded by the PC at intervals of $2^{\circ}$ of rotation. From the image-intensity (gray value) dataset of each crystal in the thin section the extinction angles of individual crystals can be determined. Similarly, eight other extinction angles of individual crystals are obtained from eight other CCD camera positions with respect to the thin section. Finally, the $c$-axis orientation of individual crystals is calculated by using these extinction angles. With this technique, all crystals within the view of the CGD camera can be analyzed at the same time. In addition, with image-processing techniques the individual crystals are recognized automatically and other parameters, such as grain-size and grain shape, can be measured simultaneously. Textural studies of Dome Fuji (Antarctica) ice cores have been conducted with this analyzer.
\end{abstract}

\section{INTRODUGTION}

The measurement of crystal-orientation fabrics and textural parameters such as crystal size and crystal elongation is imperative in order to comprehend the chemical signals from ice cores as well as the dynamical behavior of ice sheets. Continuous detailed fabric analysis along an ice core, however, has not been performed so far because the fabric analysis by conventional methods with a universal stage (Langway, 1958) is such a time-consuming and laborious process. Although several attempts (Morgan and others, 1984; Mori and others, 1985; Lange, 1988; Langway and others, 1988; Eicken, 1993; Anandakrishnan and others, 1994) at more rapid and easier measurement of ice fabric have been made over the last few decades, up to now no suitable device for automatic individual crystal recognition and orientation determination has been developed.

The main objectives of this study are to develop an automatic ice-fabric analyzer and to obtain new measurements of ice fabric and texture, which can be applied to ice-core studies. In this paper a theory of automated determination of crystal size and orientation that is based on the optical method is described. The results of textural studies on an ice core using this device are presented elsewhere in this volume (Azuma and others, 1999).

\section{METHOD AND DEVICE}

\section{Theoretical basis of the extinction method}

An individual crystal in a thin section of ice placed between a pair of crossed polaroids can be distinguished by its colour and brightness. The colour and brightness of each crystal depends on the $c$-axis orientation of the crystal. When the crossed polaroids are rotated, the brightness of each crystal

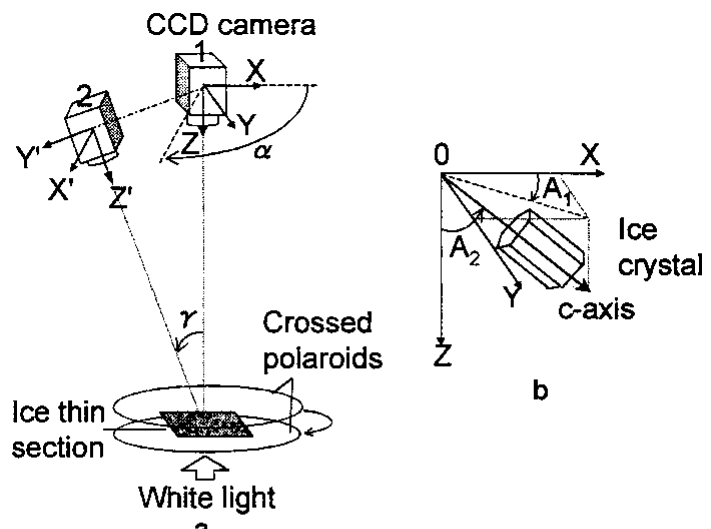

a
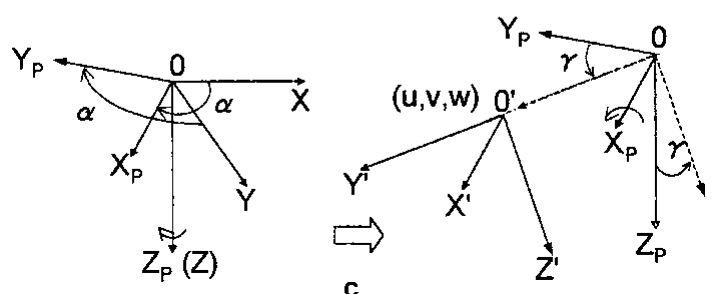

Fig. 1. Definition of c-axis orientation and camera coordinates. (a) The coordinates of camera positions; (b) the azimuth and zenith angle of a c axis; (c) the transformation of the different camera coordinates. 
changes without a change of color. A minimum brightness for an individual crystal appears at every $90^{\circ}$ interval during the polaroids rotation. This minimum-intensity position is called "extinction" and is used to determine the $c$-axis orientation by the conventional method (Langway, 1958). Using a charge coupled device (CCD) camera linked to a personal computer $(\mathrm{PC})$, we obtain four extinction angles for each individual crystal with respect to one camera position. Next we incline the camera and obtain another set of extinction angles with respect to the inclined camera position for the same crystal. With several sets of extinction angles, the $c$ axis of individual crystals can be determined as follows.

As shown in Figure la, the ice thin section is placed between the crossed polaroids parallel to the horizontal plane. With respect to a CCD camera perpendicular to the thin section, the rectangular coordinate system $X Y Z$ is taken so that the $X-Y$ plane coincides with the camera image plane and the $Z$ axis coincides with the lens principal axis. Consider the $c$ axis of a crystal as a unit vector. Its orientation is defined by azimuth $\left(A_{1}\right)$ and zenith angle $\left(A_{2}\right)$ as shown in Figure 1b. Azimuth $\left(A_{1}\right)$ is the angle between the $X$ axis and the projection of the crystal $c$ axis on the $X-Y$ plane. Zenith angle $\left(A_{2}\right)$ is the angle between the $Z$ axis and the $c$ axis. Take another coordinate system, $X^{\prime} Y^{\prime} Z^{\prime}$ with respect to the inclined camera position. This coordinate system is obtained by rotating the $X-Y$ plane $\alpha^{\circ}$ on the $Z$ axis to get $X_{\mathrm{P}} Y_{\mathrm{P}} Z_{\mathrm{P}}$ and then rotating $Y_{\mathrm{P}} Z_{\mathrm{P}}$ through $\gamma^{\circ}$ on the $X_{\mathrm{P}}$ axis (Fig. 1c). $\alpha$ and $\gamma$ are taken positive if rotated counterclockwise. Then a parallel transformation $(u, v, w)$ of $X_{\mathrm{P}} Y_{\mathrm{P}} Z_{\mathrm{P}}$ gives the system $X^{\prime} Y^{\prime} Z^{\prime}$. The transformation between $X Y Z$ and $X^{\prime} Y^{\prime} Z^{\prime}$ can be written

$$
\left(\begin{array}{c}
X_{\mathrm{P}}^{\prime} \\
Y_{\mathrm{P}}^{\prime} \\
Z_{\mathrm{P}}^{\prime}
\end{array}\right)=\left(\begin{array}{ccc}
\cos \alpha & \sin \alpha & o \\
-\sin \alpha \cos \gamma & \cos \alpha \cos \gamma & \sin \gamma \\
\sin \alpha \sin \gamma & -\cos \alpha \sin \gamma & \cos \gamma
\end{array}\right)\left(\begin{array}{c}
X_{\mathrm{P}} \\
Y_{\mathrm{P}} \\
Z_{\mathrm{P}}
\end{array}\right) \text {. }
$$

Hence, when we observe from the inclined camera a $c$ axis with $A_{1}$ and $A_{2}$ in the $X Y Z$ coordinates, the orientation of the $c$ axis is given with $A_{1}{ }^{\prime}$ and $A_{2}{ }^{\prime}$ in the $\mathrm{O}-X_{\mathrm{P}}{ }^{\prime} Y_{\mathrm{P}}{ }^{\prime} Z_{\mathrm{P}}{ }^{\prime}$ coordinates as

$$
\left(\begin{array}{c}
\sin A_{2}{ }^{\prime} \cos A_{1}{ }^{\prime} \\
\sin A_{2}{ }^{\prime} \sin A_{1}{ }^{\prime} \\
\cos A_{2}{ }^{\prime}
\end{array}\right)
$$

$=\left(\begin{array}{ccc}\cos \alpha & \sin \alpha & 0 \\ -\sin \alpha \cos \gamma & \cos \alpha \cos \gamma & \sin \gamma \\ \sin \alpha \sin \gamma & -\cos \alpha \sin \gamma & \cos \gamma\end{array}\right)\left(\begin{array}{c}\sin A_{2} \cos A_{1} \\ \sin A_{2} \sin A_{1} \\ \cos A_{2}\end{array}\right)$

From Equation (2) we obtain

$$
\begin{aligned}
& \cos ^{2} A_{1}{ }^{\prime} \\
& =\left(\cos \alpha \sin A_{2} \cos A_{1}+\sin \alpha \sin A_{2} \sin A_{1}\right)^{2} \\
& / 1-\left(\sin \alpha \sin \gamma \sin A_{2} \cos A_{1}\right. \\
& \left.-\cos \alpha \sin \gamma \sin A_{2} \sin A_{1}+\cos \gamma A_{2}\right)^{2} .
\end{aligned}
$$

If we obtain several values of $A_{1}{ }^{\prime}$ from several camera positions with different sets of $\alpha$ and $\gamma$, the values of $A_{1}$ and $A_{2}$ can be determined theoretically by Equation (3). Note that the value of $\gamma$ for the calculation is not the actual inclination angle. It must be corrected for the refraction, which occurs when light passes between crystal and air. The refraction index of ice is $n=1.31$, found from Snell's law (Kamb, 1962):

$$
n=\frac{\sin \gamma}{\sin \gamma^{\prime}},
$$

where $\gamma$ is the angle of incidence with respect to the plane of the thin section, and $\gamma^{\prime}$ is the angle of refraction in the ice. In this study, all calculations of the $c$-axis orientation with the basic Equation (3) use the corrected inclination $\gamma^{\prime}$.

At the initial position, the vibrating direction of the polarizer/analyzer is taken parallel to the $X$ axis. Extinction is obtained when the vibrating direction of either the polarizer or the analyzer coincides with the $c$ axis of individual crystals. Thus $A_{1}$ and $A_{1}{ }^{\prime}$ can be written as

$$
\begin{aligned}
& A_{1}=\theta+i 90^{\circ} \\
& {A_{1}}^{\prime}=\theta^{\prime}+i 90^{\circ} .
\end{aligned}
$$

Here $\theta$ and $\theta^{\prime}$ are the rotation angles of the polarizer/analyzer from the initial position and $i$ is one of $0,1,2$ or 3 . Equation (3) becomes

$$
\begin{aligned}
& \cos ^{2}\left(\theta^{\prime}+i 90^{\circ}\right) \\
= & {\left[\cos \alpha \sin A_{2} \cos \left(\theta+i 90^{\circ}\right)+\sin \alpha \sin A_{2} \sin \left(\theta+i 90^{\circ}\right)\right]^{2} } \\
& / 1-\left[\sin \alpha \sin \gamma \sin A_{2} \cos \left(\theta+i 90^{\circ}\right)\right. \\
& \left.-\cos \alpha \sin \gamma \sin A_{2} \sin \left(\theta+i 90^{\circ}\right)+\cos \gamma \cos A_{2}\right]^{2}
\end{aligned}
$$

We use a least-mean-squares approximation method to calculate the extinction angle from the profile of gray-value data for individual crystals with respect to the rotation of the crossed polaroids at steps of $2^{\circ}$. Using eight inclined camera positions (Table 1), the value of $i$ can be determined by comparison of the extinction angles $\left(\theta_{1}\right.$ to $\left.\theta_{8}\right)$ as shown in Table 2. In Table $1, \theta_{0}$ is equivalent to $\theta$ in Equation (6), and $\theta_{1}$ to $\theta_{8}$ are equivalent to $\theta^{\prime}$ in Equation (6). When $\theta=0^{\circ}$ or $90^{\circ}$, Equation (6) cannot give a single solution of $A_{1}$. We solve this problem by using the comparison shown in Table 3. In this case $A_{1}$ and $A_{1}{ }^{\prime}$ become:

$$
\begin{aligned}
& A_{1}=\theta \pm 45^{\circ}+i 90^{\circ} \\
& A_{1}{ }^{\prime}=\theta^{\prime} \pm 45^{\circ}+i 90^{\circ} .
\end{aligned}
$$

When the $c$ axis lies on the $X-Y$ plane $\left(A_{2}=90^{\circ}\right)$, the difference between two extinction angles in Table 2 or 3 becomes zero. In this case, we can determine the $c$-axis orientation by using the condition presented in Table 4 .

Table 1. Camera positions according to camera rotation $\alpha$ and inclination $\gamma$

\begin{tabular}{cccc}
\hline Camera position & $\gamma$ & $\alpha$ & Extinction angle \\
& $\circ$ & $\circ$ & \\
\hline & & & \\
1 & 0 & 0 & $\theta_{0}$ \\
2 & 20 & 0 & $\theta_{1}$ \\
3 & 20 & 45 & $\theta_{2}$ \\
4 & 20 & 90 & $\theta_{3}$ \\
5 & 20 & 135 & $\theta_{4}$ \\
6 & 20 & 180 & $\theta_{5}$ \\
7 & 20 & 225 & $\theta_{6}$ \\
8 & 20 & 270 & $\theta_{7}$ \\
& 20 & 315 & $\theta_{8}$ \\
\hline
\end{tabular}


Table 2. Comparison of the extinction angles to determine the value of $i$ in Equation (5)

\begin{tabular}{cll}
\hline Value of $i$ & Condition of extinction angles \\
\hline 0 & $\theta_{1}<\theta_{5}$ & and $\theta_{3}>\theta_{7}$ \\
1 & $\theta_{1}>\theta_{5}$ & and $\theta_{3}>\theta_{7}$ \\
2 & $\theta_{1}>\theta_{5}$ & and $\theta_{3}<\theta_{7}$ \\
3 & $\theta_{1}<\theta_{5}$ & and $\theta_{3}<\theta_{7}$ \\
\hline
\end{tabular}

Table 3. Comparison of the extinction angles to determine the value of $i$ in Equation (5), if $\theta$ in Equation (5) is $0^{\circ}$ or $90^{\circ}$

\begin{tabular}{|c|c|}
\hline Value of $i$ & Condition of extinction angles \\
\hline 0 & $\theta_{2}<\theta_{6}$ and $\theta_{4}>\theta_{8}$ \\
\hline 1 & $\theta_{2}>\theta_{6} \quad$ and $\theta_{4}>\theta_{8}$ \\
\hline 2 & $\theta_{2}>\theta_{6} \quad$ and $\theta_{4}<\theta_{8}$ \\
\hline 3 & $\theta_{2}<\theta_{6} \quad$ and $\theta_{4}<\theta_{8}$ \\
\hline
\end{tabular}

Table 4. Determination of the c-axis orientation normal to the vertical axis

\begin{tabular}{ccc}
\hline$A_{1}$ & $0^{\circ}<\theta_{0}<90^{\circ}$ & $\theta_{0}=0^{\circ}$ or $90^{\circ}$ \\
& \\
\hline$A_{1}=\theta_{0}$ or $\left(\theta_{0}+180^{\circ}\right)$ & $\theta_{1}=\theta_{5}<\theta_{0}$ & $\theta_{2}=\theta_{6}<\theta_{0}$ \\
$\left(\theta_{3}=\theta_{7}>\theta_{0}\right)$ & $\left(\begin{array}{c}\left.\theta_{4}=\theta_{8}>\theta_{0}\right) \\
\theta_{2}=\theta_{6}>\theta_{0} \\
\left.A_{1}=\left(\theta_{0}+90^{\circ}\right) \text { or }\left(\theta_{0}+270^{\circ}\right) \theta_{8}<\theta_{0}\right) \\
\left(\theta_{3}=\theta_{7}<\theta_{0}\right)\end{array}\right.$ \\
\end{tabular}

\section{THE AUTOMATED ANALYSIS SYSTEM}

\section{Hardware}

As shown in Figure 2, the hardware of the system consists of three sections: a measurement section, a control-box/interface and a PC. The measurement section is set up in a cold room. The device is controlled via a control-box/interface by the PC outside the cold room.

The measurement section consists of four major components: (1) a sample stage which can be rotated by a stepping motor, (2) a pair of crossed polaroids (transmissivity

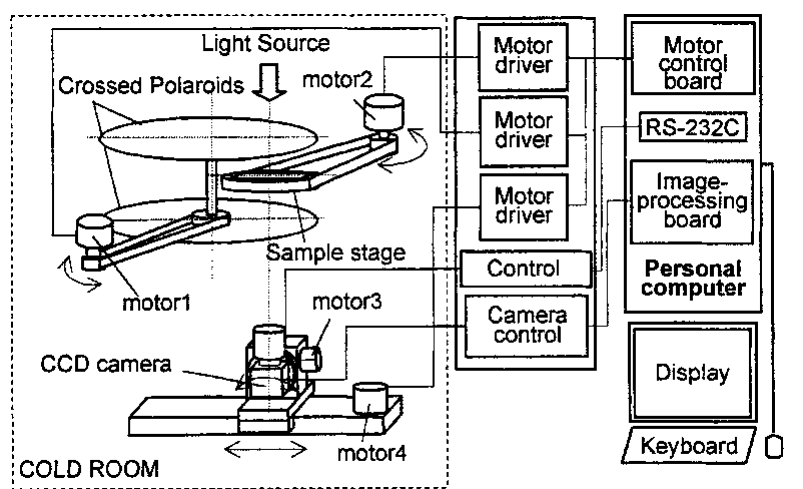

Fig. 2. Schematic diagram of automated system.
$0.0012 \%$ ) which can be rotated by a stepping motor, (3) a CCD camera (Toshiba IK-C41MF) mounted on a stepping-motor-controlled rotation stage which is mounted on a stepping-motor-controlled slide stage, and (4) a homogeneous scattered-white-light source. The rotation of the sample stage produces the rotation angle $\alpha$. The tilt angle $\gamma$ is produced by a combination of the rotation and the sliding of the CCD camera controlled by two stepping motors.

The control-box/interface comprises the motor drivers, camera control unit and power unit. A motor control board (HLS-PPD234W) and an image-processing card (Integral Technologies, FlashPoint128) are installed in the PC operated under Windows NT.

\section{Software}

In order to fulfil the automated procedures, a program performs the analysis. The program uses the functions of commercial image-processing software (Media Cybernetics: Image-Pro) to perform the image digitization and filtering operations and to measure several crystal textural parameters such as grain-size.

The procedure of the automated analysis program is shown in Figure 3a:

Step 1:

To keep measuring the same crystal from different CCD camera observation positions, a coordinate transformation between the vertical observation position and each inclined camera position is executed. Coordinate transformation indexes are saved as calibration data files. Details are described below.

Step 2:

While the crossed polaroids are rotated every $5^{\circ}$, images of samples are taken at the vertical CCD camera observation position to recognize individual crystals and finally to measure the textural parameter according to the steps shown in Figure $3 \mathrm{~b}$

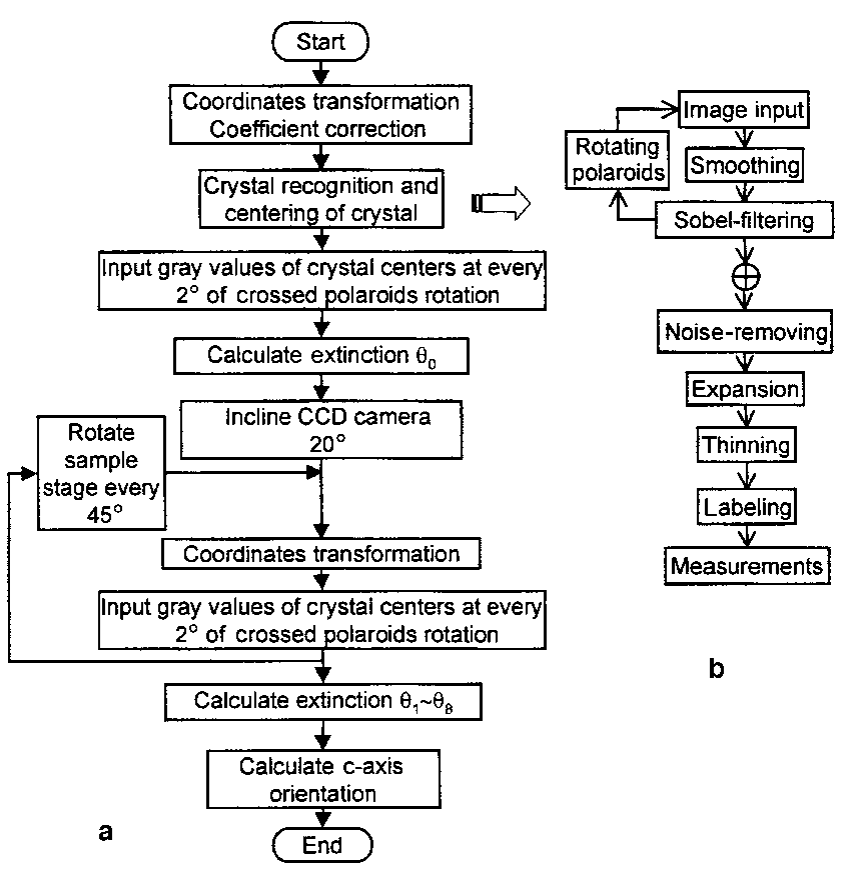

Fig. 3. Flow charts of (a) the automated fabric-analysis program and $(b)$ the pre-processing to distinguish crystals. 
Step 3:

While the crossed polaroids are rotated every $2^{\circ}$, the gray values of $3 \times 3$ pixels around the center of each crystal are measured at the vertical CCD camera observation position. The intensity data are saved as data files.

Step 4:

The CCD camera is inclined an angle of $20^{\circ}$ and moved on the slide to keep taking the same field of view $(35 \mathrm{~mm}$ diameter).

Step 5:

By using the coordinate transformation indexes obtained in step 1, coordinates of the centres of individual crystals at the inclined camera observation position are calculated.

Step 6:

The same as step 3: the gray values around the center of each crystal are measured at the inclined camera observation position.

Step 7:

The sample stage is rotated at $45^{\circ}$ intervals, between two of which steps 5 and 6 are repeated.

Step 8:

The intensity data of each crystal are approximated to a sixterm polynomial function with a least-squares error algorithm. The extinction angles of individual crystals are calculated at every CCD camera observation position by solving for the minimum of the functions over the range of $\theta$ from $0^{\circ}$ to $90^{\circ}$. Thus, for each crystal nine extinction angles, i.e. $\theta_{\mathrm{e} 0}$ to $\theta_{\mathrm{e} 8}$, are obtained.

Step 9:

The quadrant judgement is carried out by using the nine extinction angles according to Table 2, 3 or 4 for each crystal. Then the azimuth and zenith of each crystal $c$ axis are calculated.

The camera coordinate transformation is necessary in order to keep measuring the same grain at all camera positions. We consider that there are $n$ positions indicated by $\left(X_{i}, Y_{i}\right)$ $(i=1$ to $n)$ in the $\mathrm{O}-X Y Z$ coordinates. If we transform these positions into $\left(X_{i}^{\prime}, Y_{i}^{\prime}\right)$ in the $\mathrm{O}-X_{\mathrm{P}}{ }^{\prime} Y_{\mathrm{P}}{ }^{\prime} Z_{\mathrm{P}}{ }^{\prime}$ coordinates, the positions $\left(X_{i}^{\prime}, Y_{i}^{\prime}\right)$ are given by the equation

$\left(\begin{array}{c}x_{1}{ }^{\prime} \\ x_{2}{ }^{\prime} \\ \vdots \\ x_{n}{ }^{\prime} \\ y_{1}{ }^{\prime} \\ y_{2}{ }^{\prime} \\ \vdots \\ y_{n}{ }^{\prime}\end{array}\right)+\left(\begin{array}{c}e_{1 x} \\ e_{2 x} \\ \vdots \\ e_{n x} \\ e_{1 y} \\ e_{2 y} \\ \vdots \\ e_{n y}\end{array}\right)=\left(\begin{array}{cccccc}x_{1} & y_{1} & 0 & 0 & 1 & 0 \\ x_{2} & y_{2} & 0 & 0 & 1 & 0 \\ \vdots & \vdots & \vdots & \vdots & \vdots & \vdots \\ x_{n} & y_{n} & 0 & 0 & 1 & 0 \\ 0 & 0 & x_{1} & y_{1} & 0 & 1 \\ 0 & 0 & x_{2} & y_{2} & 0 & 1 \\ \vdots & \vdots & \vdots & \vdots & \vdots & \vdots \\ 0 & 0 & x_{n} & y_{n} & 0 & 1\end{array}\right)\left(\begin{array}{c}a_{11} \\ a_{12} \\ a_{21} \\ a_{22} \\ u \\ v\end{array}\right)$.

More generally,

$$
\boldsymbol{X}^{\prime}+e=\boldsymbol{X a},
$$

where $e$ is error and $\mathbf{a}$ is the transformation coefficient vector. We determine $\mathbf{a}$ by the least-mean-squares approximation method using the equation

$$
\mathbf{a}=\left(X^{T} X\right)^{-1} X^{T} X^{-1} .
$$

If we take a large number of positions (i.e. large $n$ ), the error $e$ becomes small, i.e. we can obtain a more accurate value for a. We therefore set a transparent sheet marked with 256 dots on the sample stage and input the coordinates of the dots at all camera positions in order to calculate the transformation coefficients. It was found that the accuracy of the transformation is better than $0.7 \mathrm{~mm}$ under the present magnification with $0.072 \mathrm{~mm} /$ pixel.

\section{RESULTS}

Ice-core samples from Dome F, Antarctica, have been analyzed with this system. Vertical thin sections $10 \mathrm{~cm}$ long, $5 \mathrm{~cm}$ wide and $0.5 \mathrm{~mm}$ thick were prepared, as outlined by Langway (1958). Detailed results are described elsewhere (Azuma and other, 1999). In order to verify the automated method we also measured the $c$-axis orientation fabrics of several thin sections manually by the universal stage method and compared the results with those measured automatically. Figure $4 \mathrm{a}$ and $\mathrm{b}$ show the measured $c$-axis orientation data plotted on a Schmidt net by the automated method and the universal stage method, respectively. There are several cases in which the automatic method was unable to determine a $c$-axis orientation:

(1) When the $c$ axis is nearly parallel to the $Z$ axis, the crystal remains dark while the crossed polaroids rotate at the camera position $\mathrm{O}$. As a result, the extinction angle $\theta_{0}$ cannot be determined exactly.

(2) If the crystal size is $<0.5 \mathrm{~mm}$, the accuracy of following the center of the crystal by coordinate transformation decreases. As a result, a wrong position in a neighboring crystal or on a grain boundary may be measured at different camera positions. Then Equation (6) gives no solution.

(3) When the value of $i$ in Equation (6) determined by the set of even-number extinction angles $\left(\theta_{2}, \theta_{4}, \theta_{6}, \theta_{8}\right)$ is not in agreement with that determined by the set of oddnumber extinction angles $\left(\theta_{1}, \theta_{3}, \theta_{5}, \theta_{7}\right)$, the $c$-axis orientation cannot be determined. This may be due to noise when the gray value is measured.

We have devised an algorithm to provide a null solution when the machine is faced with any of the above cases. Except where crystals fall into the above categories, the two fabric plots are in good agreement.

A sample of artificial ice with near-random $c$-axis orientations was measured by the automated system and with the universal stage. Figure 5 shows good agreement between the two fabric plots. To assess quantitatively the difference between the two measurement techniques, the angular difference between the $c$-axis orientations measured with the automated system and those measured with the universal stage was calculated for every crystal in the two artificial ice samples. A histogram of the $c$-axis misorientation angles is shown in Figure 6. It is seen that a misorientation angle of about $5^{\circ}$ is predominant. The reason is discussed below.

\section{ACGURAGY OF THE $c$-AXIS ORIENTATION MEA- SUREMENT OF THE AUTOMATED SYSTEM}

One way of assessing the accuracy of the automated analysis is to investigate the reproducibility. Five times, we measured the $c$-axis orientations of 220 crystals within the two samples referred to above and calculated the resultant vector for 

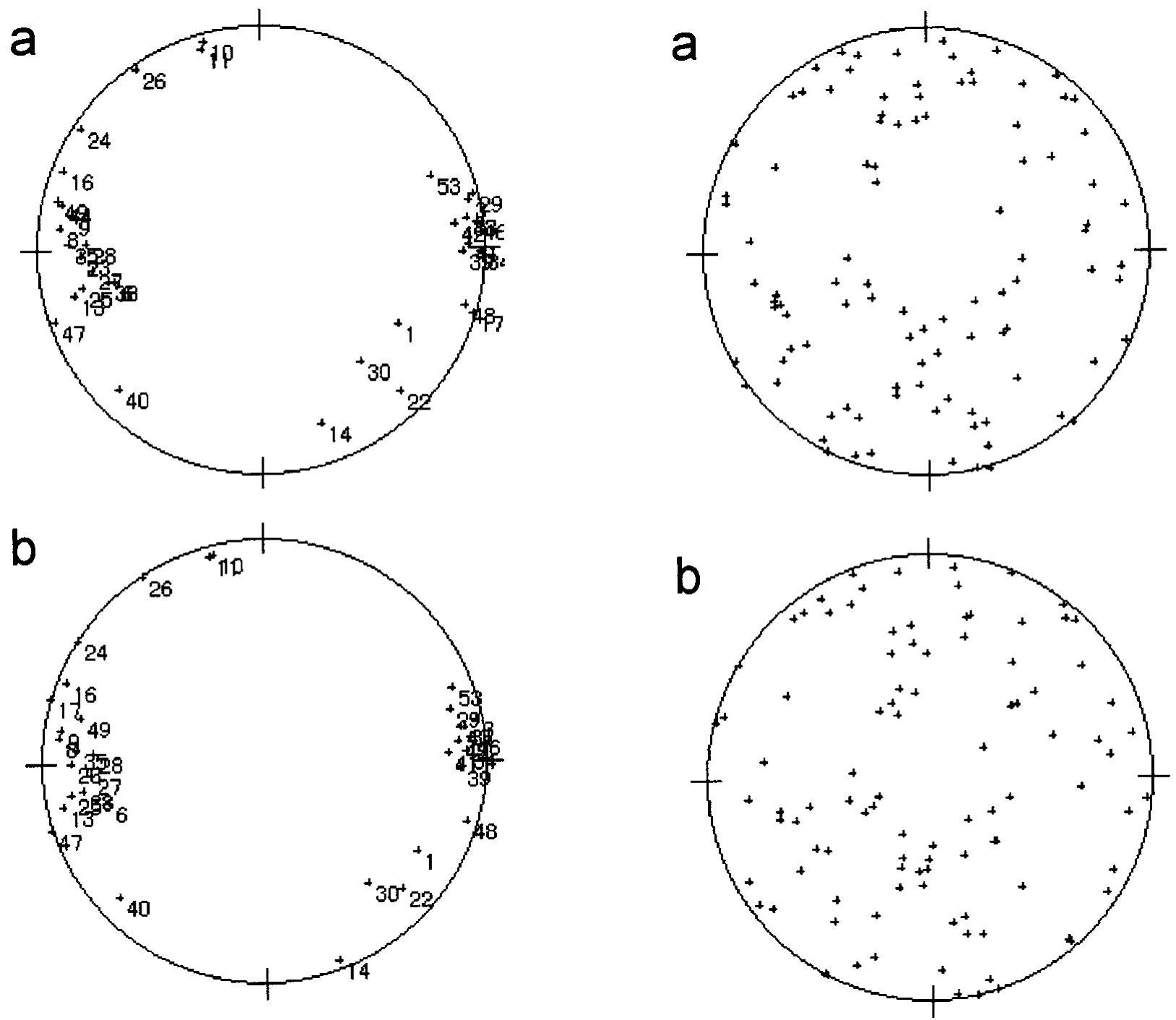

Fig. 4. Fabric diagrams on a vertical thin section from Dome Fuji ice core at $1800 \mathrm{~m}$ depth (a) by the automated method and $(b)$ by the universal stage method. The number by each dot designates each crystal's label number.

each crystal, which is obtained by summing all five $c$-axis unit vectors. The deviation angle from the orientation of the resultant vector was obtained for each $c$-axis measurement. The histogram of the deviation angles is shown in Figure 7. It was found that the reproducibility is better than $1^{\circ}$.

In addition, the accuracy of the automated system can be evaluated by the accuracy of each value of azimuth and tilt, because the $c$-axis orientation is defined by azimuth and tilt. As stated above, the azimuth is determined from the extinction angle obtained by the vertically positioned camera. Therefore, the errors in azimuth measurement are the errors in the extinction-angle measurement, which mainly result from the fluctuation and instability of the crossed-polaroids rotation motor, gray-value measurement, and approximate solution of the extinction angle from the grayvalue data profile. The accuracy provided by the manufacturer for the motor rotation angle is within $0.05^{\circ}$. However, the fluctuation of the gray values caused by bubbles, noises and camera and light-source irregularity is difficult to estimate. Since we use the bisection method on the meansquare approximation curve of gray values to solve the minimum/maximum gray value, the extinction angle can be determined accurately without being affected by the fluctuation of the gray values. We cannot know all the inaccura-

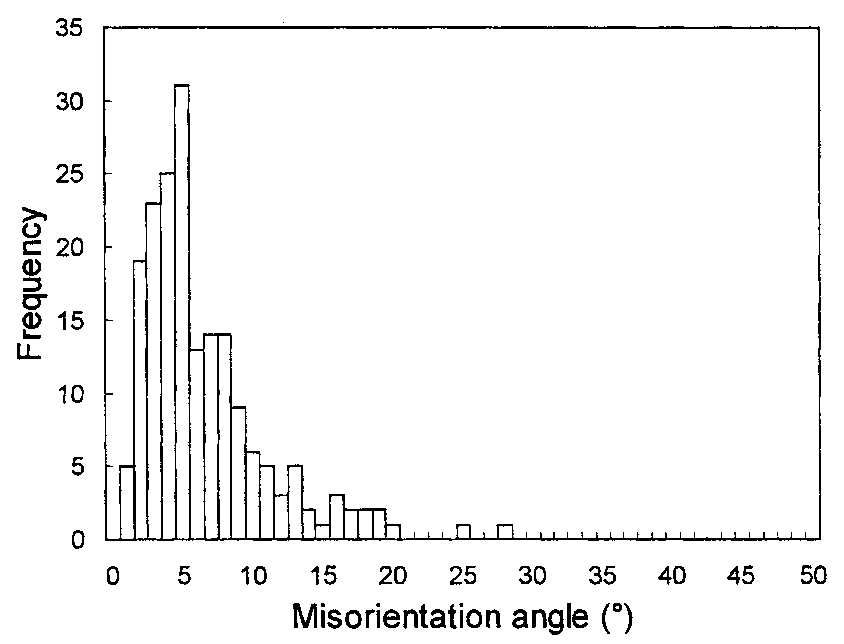

Fig. 6. Histogram of the misorientation angle between the $c$ axis orientations obtained by the automated method and by the universal stage method.

Fig. 5. Fabric diagrams on an artificial-ice thin section (a) by the automated method and (b) by the universal stage method.

cies of the above components contributing to errors in azimuth measurement. Further, the components are not independent of each other. As these errors can be considered as random, the estimate we obtain of total error by repeat measurements is empirical. In the reproducibility experi- 


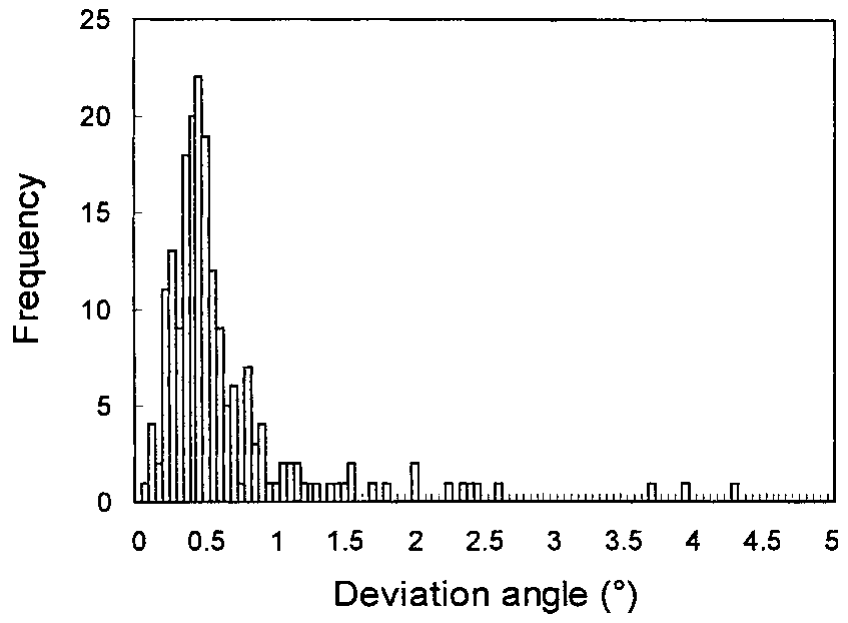

Fig. 7. Histogram of the reproducibility of the results by the automated method.

ment, the standard deviation $\bar{s}$ of the five azimuths for each crystal was calculated as $0.17^{\circ}$. Generally, we quote $\pm 2 \bar{s}$ as the measurement error, i.e. a single measurement has a $95 \%$ probability of lying within $\pm 2 \bar{s}$ of its average result. Thus, we can say the accuracy of the azimuth is $\pm 0.34^{\circ}$.

On the other hand, since the zenith is a computed quantity, we cannot use the same method to obtain the inaccu-

a

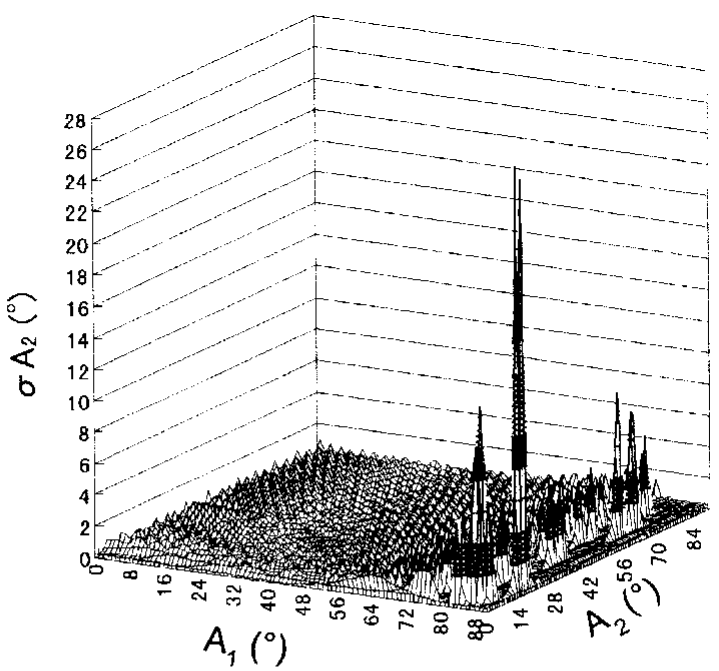

b

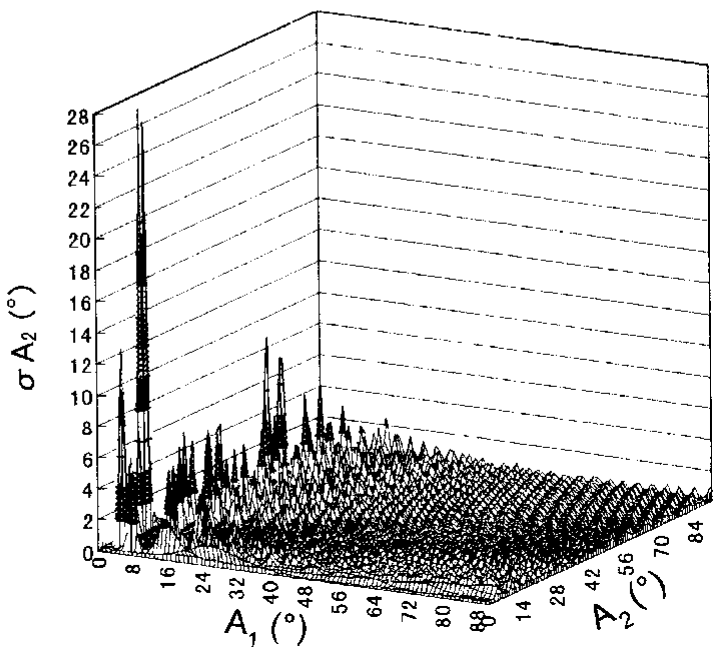

Fig. 8. Calculated errors $\sigma A_{2}$ using $A_{1}{ }^{\prime}$ obtained (a) by the inclined camera position 1 , and $(b)$ by the inclined camera position 3. racy of the azimuth (extinction angle). The zenith $A_{2}$ is a function of azimuth $A_{1}$, azimuth $A_{1}{ }^{\prime}$ observed from the inclined camera position, stage rotation $\alpha$ and camera inclination $\gamma$. That is,

$$
A_{2}=f\left(A_{1}, A_{1}^{\prime}, \alpha, \gamma\right)
$$

The inaccuracy of $A_{2}$ expressed as $\alpha A_{2}$ can be obtained by the root-sum-square formula

$$
\begin{gathered}
= \pm\left[\left(\frac{\partial f}{\partial A_{1}}\right)^{2}\left(\sigma A_{1}\right)^{2}+\left(\frac{\partial f}{\partial A_{1}{ }^{\prime}}\right)\left(\sigma A_{1}^{\prime}\right)^{2}\right. \\
\left.+\left(\frac{\partial f}{\partial \alpha}\right)(\sigma \alpha)^{2}+\left(\frac{\partial f}{\partial \gamma}\right)^{2}(\sigma \gamma)^{2}\right]^{\frac{1}{2}},
\end{gathered}
$$

where $\sigma A_{1}, \sigma A_{1}{ }^{\prime}, \sigma \alpha$ and $\sigma \gamma$ are the errors in $A_{1}, A_{1}{ }^{\prime}, \alpha$ and $\gamma$, respectively. $\sigma A_{1}$ can be taken as $\pm 2 \bar{s}$ obtained above. Similarly, $\sigma A_{1}{ }^{\prime}$ can be considered the same as the error in the extinction angle observed from the inclined camera position. As for $\sigma \alpha$, it comes from the error of the stage rotation motor provided by the manufacturer, i.e. $\pm 0.05^{\circ}$. The error of camera inclination $\sigma \gamma$ is due to the inaccuracy of the inclination motor, $\pm 0.2^{\circ}$. Let us consider here only the case of a $c$ axis which exists in the first quadrant $\left(0^{\circ} \leq A_{1}<90^{\circ}\right)$, because a similar result can be obtained in other quadrants. Using Equations (3), (11) and (12), we calculate the error $\sigma A_{2}$ of the zenith angle $A_{2}$ with various sets of $A_{1}$ and $A_{2}$. Figure $8 \mathrm{a}$ and $\mathrm{b}$ are the calculated results using the $A_{1}{ }^{\prime}$ value obtained by the inclined camera positions 1 and 3 , respectively. Where camera position 1 is used, $\sigma A_{2}$ is $<2^{\circ}$ over the $A_{1}$ range smaller than $70^{\circ}$. Where camera position 3 is used, we can make $\sigma A_{2}<2^{\circ}$ over the $A_{1}$ range larger than $20^{\circ}$. In other words, by choosing the most suitable inclined camera position to obtain $A_{1}{ }^{\prime}$ we can reduce the error $\sigma A_{2}$ of the zenith angle $A_{2}$ to within $2^{\circ}$ for any range of $A_{1}$ and $A_{2}$

Compared with the accuracy of the automatic device $\left(\sigma A_{1} \leq \pm 0.34^{\circ}\right.$ and $\left.\sigma A_{2} \leq \pm 2^{\circ}\right)$ stated above, the accuracy of the universal stage is considered to be $\pm 5^{\circ}$ (Langway, 1958). However, the error in the universal stage method is related to the skill of the observer and would be much more than $\pm 5^{\circ}$ in some cases. This may account for the misorientation of $10^{\circ}$ or $20^{\circ}$ evident in Figure 6. In addition, reproducibility when using the universal stage is usually $1-2^{\circ}$, compared with $<1^{\circ}$ for the automatic device. It is concluded that the automatic device is more accurate than the universal stage.

\section{VISUALIZATION OF FABRIG AND TEXTURAL DATA}

The automated fabric-analysis system can determine individual $c$-axis orientations in a thin section rapidly, regardless of the number of crystals. Data are presented for individual crystal specifications ( $c$-axis orientation, crystal size, aspect ratio and crystal-elongation direction) and for bulk thin-section data (Schmidt diagram, median inclination, mean crystal size and mean aspect ratio). In order to investigate regional characteristics of fabric and texture, we propose a method of visual presentations of the thin-section crystal image. The method is to "colorize" the image according to the data, i.e. pseudo-color the image. For example, the $c$-axis orientation of each crystal is indicated by a pair of colors. The azimuth angle is given by one color hue that changes as 


\section{a) Azimuth}

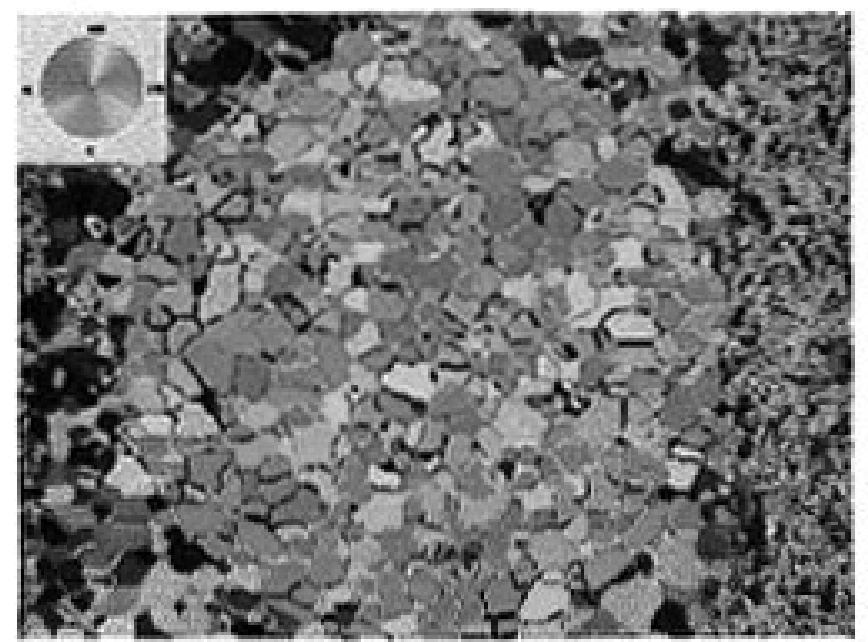

c) Schmid factor

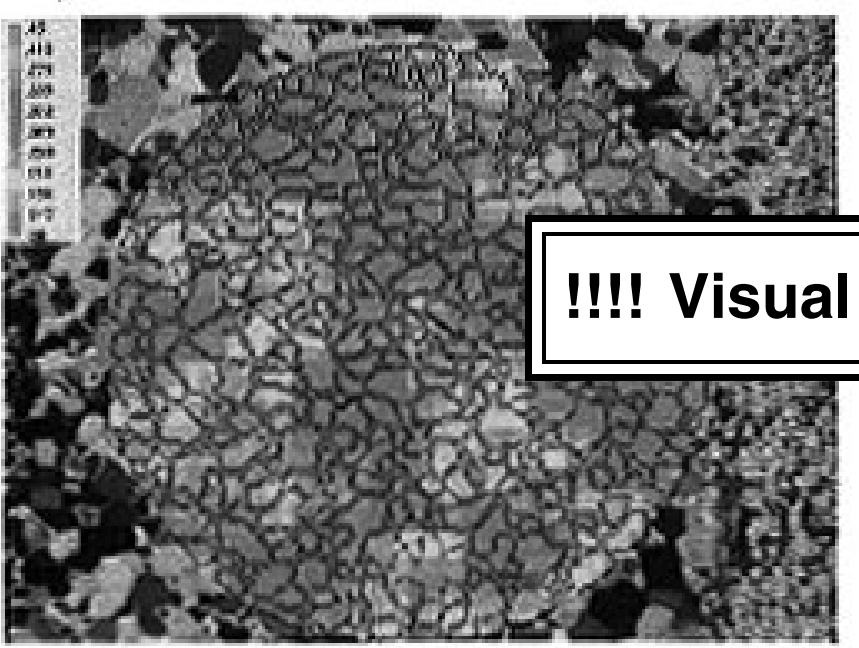

\section{e) Aspect ratio}

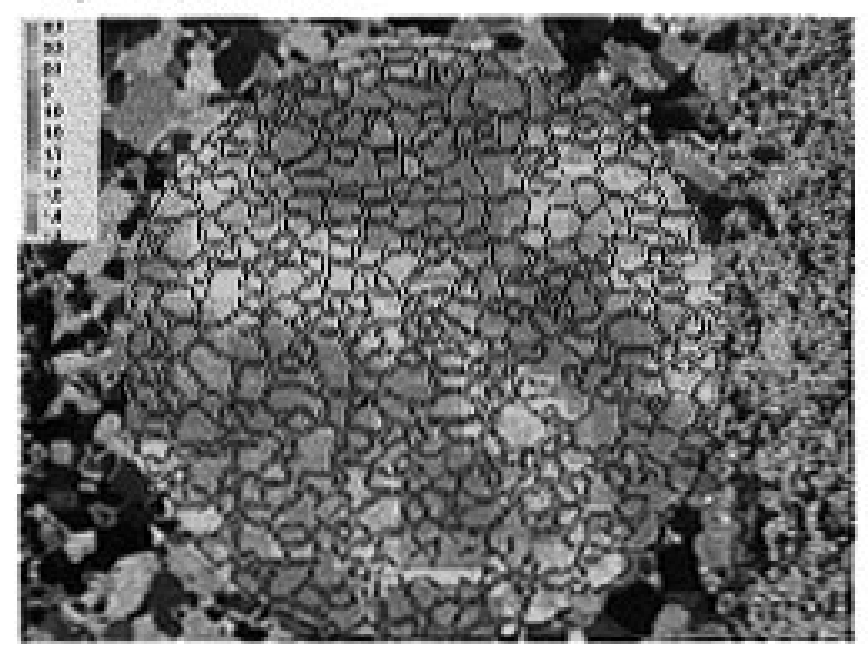

b) Zenith

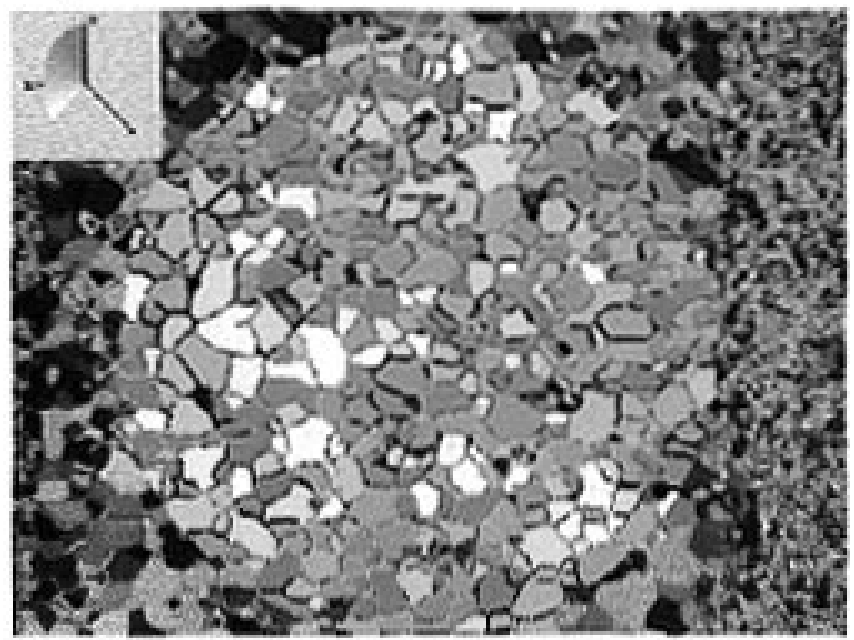

d) Grain size

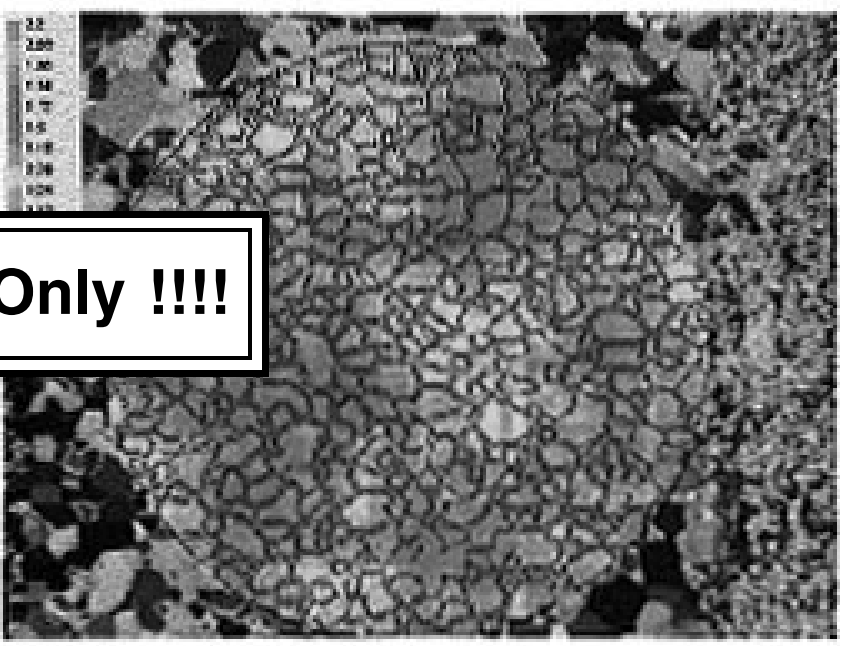

f) Elongated direction

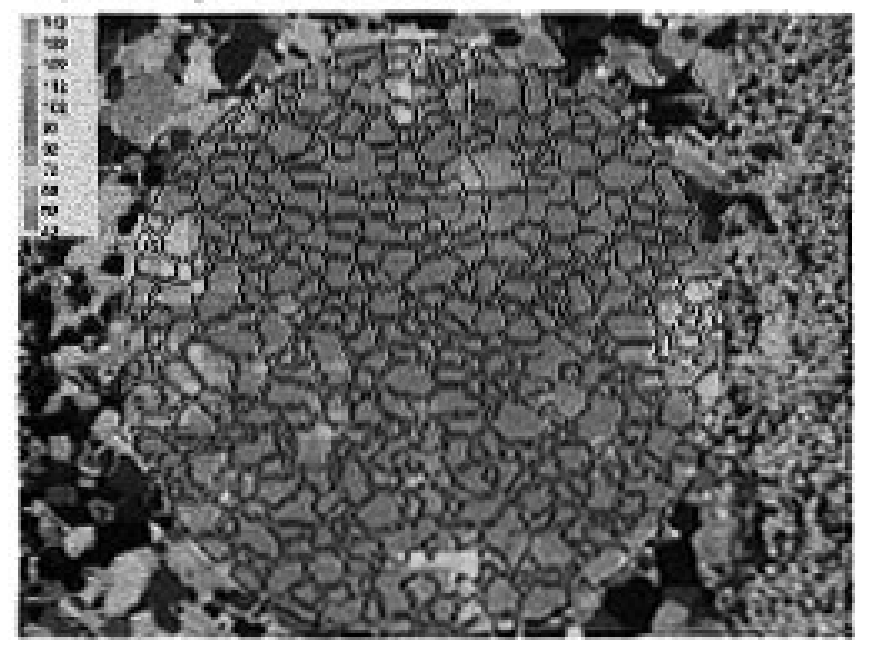

Fig. 9.Visual presentation on the thin-section crystal image: (a) azimuth; (b) zenith angle; (c) local mean Schmidt factor; (d) local mean grain-size; (e) local mean aspect ratio; $(f)$ local mean direction of crystal elongation.

shown in Figure 9a, while the zenith angle is given by the intensity of a color as shown in Figure 9b. The azimuth range $\left(0-360^{\circ}\right)$ is divided into the number of intervals specified (here the division is $5^{\circ}$ ), and each interval is assigned to a color. The color palette on the left corner represents the entire azimuth. Similarly, in Figure $9 \mathrm{~b}$ the zenith-angle range $\left(0-90^{\circ}\right)$ is divided into 90 intervals, and each interval is as- signed a specified intensity of blue color. The crystal with darker tone shows the more parallel $c$-axis alignment to the core axis. With this presentation we can see visually individual crystal orientations, local differences in crystal orientation and the relation between crystal shape and crystal orientation. Figure $9 \mathrm{c}-\mathrm{f}$ show the local mean Schmidt factor, the local mean grain-size, the local mean aspect ratio and 
the local mean crystal-elongation direction. The local area is defined as an area in which ten crystals are included. In this figure the Schmidt factor is defined as

$$
S=\sin \phi \cos \phi,
$$

where $\phi$ is the angle between each $c$ axis and the core axis. Hence, Figure $9 \mathrm{c}$ indicates that the red color region can deform easily with respect to the axial compression, while the blue colour region is hard to deform. Aspect ratio is the ratio of the major axis to the minor axis of the ellipse equivalent to the crystal on the thin-section plane. The crystalelongation direction is the angle between the core axis and the major axis of the ellipse equivalent to the crystal. The local mean Schmidt factor, the local mean grain-size, the local mean aspect ratio and the local mean crystal-elongation direction defined above are calculated over the area equivalent to ten times the mean crystal area and are plotted on a $10 \times 10$ pixel grid centered on the area. All pixels in a grid are then painted the same color. This software is installed in the present automated system.

\section{CONGLUSION}

An automatic ice-fabric analyzer using image-analysis techniques has been developed. This system enables us to measure rapidly not only $c$-axis orientations of individual crystals but also individual crystal size/shape on a thin section. Fabricaccuracy analysis indicates that the automatic device is more accurate than the universal stage, with an error of $A_{1} \leq \pm 0.34^{\circ}, A_{2} \leq \pm 1^{\circ}$ and reproducibility $<1^{\circ}$. A disadvantage of this method is that if the $c$ axis is near-parallel to the optical axis ( $Z$ axis) the $c$-axis orientation cannot be determined exactly by this method (error $\leq 10^{\circ}$ ). Therefore, in the case of an ice-core sample in which most of the $c$ axes align to the core axis, we need to avoid this condition by not taking horizontal (perpendicular to the core axis) thin sections.

\section{ACKNOWLEDGEMENTS}

An initial attempt to develop a new, rapid method of ice-fabric analysis upon which the present study is based was made by one of the authors (N.A.) at the Ice Core Laboratory, State University of New York-Buffalo in 1986. The authors especially thank C. C. Langway, Jr and H. Shoji for giving N.A. this opportunity and for valuable discussions. We are indebted to Y. Maruhashi, H. Hara, H. Yamaguchi and T. Nakajima who helped to conduct experimental studies to develop this method. We are grateful to O. Watanabe, $\mathrm{H}$. Narita, M. Nakawo, S. Fujita, T. Hondoh, K. Goto-Azuma, V. Morgan, T. H. Jacka, LiJun and an anonymous reviewer for helpful comments. This work was supported by a grantin-aid for scientific research from the Ministry of Education, Culture and Science of Japan.

\section{REFERENGES}

Anandakrishnan, S., J. J. FitzPatrick, R. B. Alley, A. J. Gow and D. A. Meese. 1994. Shear-wave detection of asymmetric $c$-axis fabrics in the GISP2 ice core, Greenland. f. Glaciol., 40 (136), 491-496.

Azuma, N. and 6 others. 1999. Textures and fabrics in the Dome F (Antarctica) ice core. Ann. Glaciol., 29 (see paper in this volume).

Eicken, H. 1993. Automated image analysis of ice thin sections - instrumentation, methods and extraction of stereological and textural parameters. F. Glaciol., 39(132), 341-352.

Kamb, W. B. 1962. Refraction corrections for universal stage measurements. I. Uniaxial crystals. Am. Mineral., 47(3), 227-245.

Lange, M. A. 1988. A computer-controlled system for ice-fabric analysis on a Rigsby stage. Ann. Glaciol., 10, 92-94.

Langway, C. C., Jr. 1958. Ice fabrics and the universal stage. SIPRE Tech. Rep. 62.

Langway, C. C., Jr, H. Shoji and N. Azuma. 1988. Crystal size and orientation patterns in the Wisconsin-age ice from Dye 3, Greenland. Ann. Glaciol., 10, 109-115.

Morgan, V. I., E. R. Davis and E. Wehrle. 1984. A Rigsby stage with remote computer compatible output. Cold Reg. Sci. Technol., 10(1), 89-92.

Mori, Y., T. Hondoh and A. Higashi. 1985. Development of an automatic ice fabric analyser. Ann. Glaciol., 6, 281-283. 